\title{
INNOVACIÓN EDUCATIVA Y PRÁCTICA PEDAGÓGICA DOCENTE EN INSTITUCIONES EDUCATIVAS RURALES EN EL PERÚ EN TIEMPOS DE PANDEMIA
}

\author{
Edgar L. Martínez-Huamán* \\ https://orcid.org/0000-0002-3839-7723 \\ Edwin Daniel Félix Benites** \\ https://orcid.org/0000-0001-8690-354X \\ Rolando Alfredo Quispe Morales*** \\ https://orcid.org/0000-0003-3140-8968
}

RECIBIDO: Junio 2021 / ACEPTADO: Octubre 2021 / PUBLICADO: Enero 2022

\begin{abstract}
Como citar: Martínez-Huamán, Edgar; Félix Benites, Edwin; Quispe Morales, Rolando. (2022). Innovación educativa y práctica pedagógica docente en instituciones educativas rurales en el Perú en tiempos de pandemia. Telos: revista de Estudios Interdisciplinarios en Ciencias Sociales, 24 (1), Venezuela. (Pp. 62-78).

DOI: www.doi.org/10.36390/telos 241.05
\end{abstract}

\section{RESUMEN}

El estudio tuvo el objetivo de comprender las percepciones de los docentes acerca de la innovación y la práctica pedagógica en instituciones educativas rurales en el Perú en tiempos de pandemia. El estudio consideró a los docentes de las instituciones educativas rurales de las diferentes provincias de la región de Ayacucho, Perú. El estudio corresponde al enfoque mixto de nivel exploratorio. Los datos cuantitativos fueron operacionalizados por un cuestionario por Google forms y lo cualitativo fue aportado por las entrevistas por medio de un guión a través de Zoom, los cuales fueron grabadas; la información se vincula mediante un enfoque interpretativo. Las categorías que emergieron fueron: adaptación a la innovación educativa, formación docente, apoyo familiar, nuevos roles de los docentes y estudiantes. Emergieron puntos de vistas favorables de los docentes que laboran en instituciones rurales en esta región del país, quienes se adaptaron a la educación bajo la modalidad virtual, asumiendo la necesidad de aprender, autoformarse y actualizarse para el manejo de los equipos y las aplicaciones para hacer su práctica pedagógica destacando que aún persisten muchas debilidades en la enseñanza virtual, sin discutir las bondades que ha traído su apoyo al proceso de aprendizaje de los educandos. Por lo que es necesario desarrollar secuencias centradas en las TIC para la capacitación,

\footnotetext{
* Doctor en Educación. Docente principal. Universidad Nacional José Marìa Arguedas. Perú. Correo electrónico: emartinez@unajma.edu.pe

** Magíster en Ciencias de la Educación. Docente asociado de la Universidad Nacional José María Arguedas. Perú. Correo electrónico: efelix@unaima.edu.pe

*** Doctor en Ciencias de la Educación. Docente del área de investigación educativa. Universidad Nacional de San Cristóbal de Huamanga.Perú. Correo electrónico: rolando.quispe@unsch.edu.pe
} 
planificación gestión y disposición de recursos con el objeto de ofrecer mejores experiencias educativas.

Palabras claves: Educación rural, innovación pedagógica, adaptación, cambios, formación docente, apoyo familiar, roles, competencias digitales.

\title{
Educational innovation and pedagogical practice in rural educational institutions in Peru on times of pandemic
}

\begin{abstract}
The study aimed to understand teachers' perceptions about innovation and pedagogical practice in rural educational institutions in Peru in times of pandemic. The study considered teachers from rural educational institutions in different provinces of Ayacucho region, Perú. The study corresponds to the mixed approach of exploratory level. The quantitative data were operationalized by a questionnaire through Google forms and the qualitative data was contributed by the interviews through a script by means of Zoom, which were recorded; the information is linked by means of an interpretative approach. The categories that emerged were: adaptation to educational innovation, teacher training, family support, new roles of teachers and students. Favorable points of view emerged from teachers working in rural institutions in this region of the country, who adapted to education under the virtual modality, assuming the need to learn, selftrain and update themselves for the management of equipment and applications to carry out their pedagogical practice, emphasizing that many weaknesses still persist in virtual teaching, without discussing the benefits that its support has brought to the learning process of students. Therefore, it is necessary to develop sequences focused on ICT for training, planning, management and provision of resources in order to offer better educational experiences.
\end{abstract}

Keywords: Rural education, pedagogical innovation, adaptation, changes, teacher training, family support, roles, digital competencies.

\section{Introducción}

La emergencia sanitaria se declaró en todo el mundo por la propagación del coronavirus (COVID-19), el número de infectados coloco en alerta a todas los ámbitos: político, salud, educativo y económico, por lo que se decretó el aislamiento social, como medida de freno al contagio (OMS, 2020; Hernández Barrios et al., 2021). Por lo que la sociedad se vio circunscrita a una gran calamidad que ha marcado la historia, por la gran cantidad de muertes e impacto psicológicos en la población causado por una de las pandemias más devastadoras que ha sufrido hasta el momento la humanidad (Méndez Calderón, 2021). Este nuevo escenario mundial impacto especialmente a la salud y a la educación (Neira-González \& Pulgarin, 2021).

El Ministerio de Educación del Perú, estableció desarrollar el servicio educativo a distancia para garantizar la atención en las instituciones de Educación Básica Regular durante el año 2020 y 2021, a través de la estrategia "Aprendo en Casa" (Estrada Araoz y Mamani Roque, 2021). La adaptación de los docentes al empleo de herramientas tecnológicas, así como la introducción de nuevas maneras de realizar la práctica pedagógica permitieron la continuidad del servicio educativo. 
Innovación educativa y práctica pedagógica docente en instituciones educativas rurales en el Perú en tiempos de pandemia

Es necesario realizar una mirada desde la disrupción educativa que existe frente a la aparición de la pandemia que ha provocado un cambio profundo e inmediato en los sistemas educativos de todos los niveles, trayendo consigo, de manera urgente, transformaciones profundas; por ello, en tiempos tan inciertos, desafiantes y cambiantes, se requiere guiar tal transformación digital en los contextos educativos (Romero Escalante, 2021; Watermeyer et al., 2021), que han sido dificultosos sobre llevar el cambio de la práctica pedagógica de ambientes presenciales a virtuales mediante las TICS a los espacios urbanos, aún más ha costado en el contexto rural (Peirano et al., 2015).

Por tanto, es relevante señalar que la presencia del empleo de las TICS en el ámbito escolar, tanto urbano como rural, trae como consecuencia una nueva definición de roles y funciones de todos los integrantes de la comunidad educativa porque la educación a distancia involucra la disponibilidad de plataformas virtuales, contenidos digitales y una formación pedagógica adecuada de los docentes, siendo un desafío realizar una práctica pedagógica en este contexto (Orozco-Cazco, et al., 2020; Sandia et al., 2018).

Para los países latinoamericanos, la innovación educativa en tiempos de pandemia es un tema completamente desafiante, en cuanto a conectividad, empleo de recursos tecnológicos y el acompañamiento activo de las familias para la formación de los educandos. Existen muchos autores que han aportado a la definición de la innovación educativa. Moreno-Correa (2020), la define como "una serie de actuaciones, decisiones y procesos, con cierto grado de pretensiones y sistematizaciones, que tratan de cambiar actitudes, contenidos, modelos, prácticas pedagógicas, ideas y culturas" (p.17). La innovación educativa está relacionada con la introducción de ideas nuevas, propuestas novedosas para la solución de problemas dentro del hacer escolar.

La innovación educativa surge de las necesidades en el contexto escolar, la nueva realidad ha contribuido a transformar el escenario a nivel macro y micro en función de desarrollar la actividad pedagógica y darle continuidad al proceso de enseñanza aprendizaje (Ramírez, 2020). De allí que la innovación sea un proceso intencional cuyo objeto es mejorar los procesos de enseñanza aprendizaje, la praxis pedagógica, con carácter sostenible cuyos resultados sean transferibles a otros contextos. Por eso, cuando se aborda la innovación educativa se debe incluir los conceptos de cambio y mejora (Pila Martínez et al., 2020).

Para Sein-Echaluce et al. (2016), la innovación educativa es la aplicación de una idea que produce un cambio planificado en los procesos, servicios o productos y que generan mejora en los procedimientos formativos. Es relevante que los educadores puedan facilitar a los educandos las innovaciones propuestas en el proceso de enseñanza aprendizaje con el fin de poder identificar el impacto generado con lo nuevo mediante el proceso planteado por la innovación. Con el arribo de las TIC a los contextos escolares en la década de los 80 , ha sido una palanca de mejora y a la vez otorga un nuevo impulso a los métodos pedagógicos, propiciando en el sistema escolar la búsqueda de nuevos caminos innovadores en el proceso formativo (Islas, 2018).

Asimismo, en las actuales circunstancias por la que atraviesa el sistema educativo, la práctica pedagógica tiene real relevancia por tratarse de un espacio donde convergen todas las acciones y esfuerzos que realiza el docente para lograr mejores aprendizajes en los educandos. Además, la práctica pedagógica, como actividad en el aula que desarrolla el docente, tiene como 
finalidad la formación integral del estudiante de acuerdo a un currículo establecido (Loaiza Zuluaga et al., 2012). La ejecución de acciones que realiza el docente en la práctica pedagógica está relacionadas al uso de estrategias de enseñanza aprendizaje, planificación curricular, aplicación de la didáctica y metodología, elaboración y empleo de materiales educativos, evaluación de los aprendizajes y el trabajo con la comunidad educativa, todo ello se adquiere por medio de la formación docente continua o permanente (Torres-González, 2021).

Por lo que se considera que los docentes deben estar actualizados y bien formados en competencias digitales. Estudios anteriores, como el de Parra y Méndez (2005), señalan que la práctica pedagógica y la educación virtual en el contexto rural debe superar las limitaciones de la tecnología dado que se genera un nuevo entorno virtual de aprendizaje que exige que los docentes y educandos ajusten las maneras de participar y relacionar dentro del proceso formativo. El docente dentro de su hacer pedagógico bajo estas condiciones de educación virtual, online 0 a distancia, recurre a un conjunto de actividades y acciones las cuales deben utilizar la didáctica y la pedagogía para brindar una adecuada formación educativa de acuerdo a la realidad del educando (Basto-Torrado, 2011).

Como se puede observar la educación virtual en el contexto rural no es nada nuevo, si bien es cierto ha tenido más tropiezos en comparación con otros contextos escolares urbanos, sin embargo, existe una gran mayoría de docentes que no se han certificado en competencia digital, desconociendo metodologías como medios para el desarrollo de las clases virtuales (Vásquez-Ponce et al., 2020). La apropiación de competencias digitales demuestran la incidencia positiva en la práctica pedagógica a través de la innovación educativa tal como lo refleja Hernández Pino (2015), al considerar que mientras se realice cosas distintas en el aula con el empleo de la TICS, estas facilitaran el fomento y desarrollo de habilidades para la solución de los problemas, la socialización y el compartir de experiencias para el aprendizaje continuo y mejora de las condiciones de vida de los actores involucrados.

Por otra parte, se puede decir, que son escasos los estudios con docentes que laboran en contextos rurales en educación primaria en tiempos de pandemia, por lo que este estudio constituye un referente para aproximar la realidad que viven el escenario educativo en esta zona del Perú. Teniendo en cuenta que las instituciones educativas rurales en su mayoría son multigrados, donde se desarrolla una pedagogía inclusiva, reconociendo los ritmos e individualidades del aprendizaje de los educandos, que convoca hoy más que nunca que los docentes se organicen en comunidades de aprendizaje en función al empleo de tecnologías disponibles (Peirano et al., 2015) para ofrecer una educación integral y adecuada a los educandos.

De esta manera es pertinente conocer y comprender las percepciones del docente de escuelas rurales de la región de Ayacucho, Perú en su práctica pedagógica con relación a la innovación educativa provocada por la pandemia. Dentro de este marco, la innovación educativa que realiza el docente dentro del aula tiene como finalidad mejorar los procesos de aprendizaje, aplicando nuevas y variadas formas de trabajo. Por eso, cualquier sistema educativo tiene que facilitar y enfatizar el desarrollo de una transformación de la práctica pedagógica de los docentes como respuesta de la institución educativa con las exigencias del entorno social y tecnológico (Vila Pérez y Núñez García, 2020).

Con ello, este estudio se enmarcó en un enfoque mixto de nivel exploratorio, que, para Hernández-Sampieri y Mendoza (2018), los estudios mixtos son sistemáticos, críticos y 
Innovación educativa y práctica pedagógica docente en instituciones educativas rurales en el Perú en tiempos de pandemia

empíricos que implican la obtención y análisis de información cuantitativa y cualitativa, discutida de manera integrada para su mejor comprensión (p.546). Desde la perspectiva cualitativa se fundamentó en la hermenéutica de Heidegger (2003), para quien el lenguaje y los hechos es la casa del ser, la interpretación del ser y Gadamer (1993), quien señala que el diálogo es una rica experiencia e induce a cambios en las personas que las vive. Dado que la cuestión principal fue conocer ¿Cuáles son las percepciones de los docentes que laboran en contextos rurales acerca de la innovación y la práctica pedagógica en la educación a distancia en tiempos de pandemia?

En la parte cuantitativa se les aplicó una encuesta a 28 docentes de escuelas rurales de diferentes provincias de la región de Ayacucho (Tabla 1), quienes estudian la Maestría en Gestión Educacional en la Universidad Nacional San Cristóbal de Huamanga, cuyo cuestionario comprendió 12 ítems, con tres dimensiones: innovación por emergencia, innovación para la mejora de los aprendizajes e innovación para la mejora de la práctica docente, por lo que fue validado por especialistas. Posteriormente se muestra el resultado de la encuesta y seguidamente las entrevistas que complementan y enriquecen la información del estudio. La muestra fue intencional, 12 hombres y 16 mujeres, muestra que se encontraba al alcance de los investigadores. El criterio de selección fue a) ser docente con más de ocho años de experiencia docente en el área rural, b) interesado en participar en el estudio, c) trabajar en aula, d) tener computador o telefonía móvil. Y en la parte cualitativa se empleó entrevistas semiestructuradas que permitió conocer las percepciones de los docentes participantes, acercándose así a la realidad ocurrida en el contexto.

Tabla 1. Docentes zona rural de la Región de Ayacucho

\begin{tabular}{|c|c|c|c|}
\hline $\mathbf{N}^{0}$ & Instituciones Educativas & Zonas rurales & Docentes \\
\hline 1 & $\begin{array}{l}\text { I.E. N²432-59/Mx-M Yanamilla- } \\
\text { Tambillo }\end{array}$ & Huanta & 3 \\
\hline 2 & I.E. $N^{\circ} 38245$ & Pallccacancha & 3 \\
\hline 3 & I.E. "Ciro Alegría" & Accomarca - Vilcas Huamán & 2 \\
\hline 4 & I.E. "Haya de la Torre" & Vincho & 3 \\
\hline 5 & I.E. "Mariscal Andrés Avelino Cáceres" & Paico-Sucre & 2 \\
\hline 6 & I.E. "Tomas Gamarra" & Acocro - Matará & 3 \\
\hline 7 & I.E. N 38705 & Samugari & 3 \\
\hline 8 & $\begin{array}{l}\text { I.E. No 432-74/Mx-M "Nuestra Señora } \\
\text { Concebida de Acco" }\end{array}$ & Acocro - Acco & 3 \\
\hline 9 & Chuschi-Quispillaqta & Chuschi - Quispillaqta & 3 \\
\hline \multirow[t]{2}{*}{10} & I.E. N ${ }^{\circ} 38405 / \mathrm{Mx}$ Osqococha & La Mar-Anco & 3 \\
\hline & & Total & 28 \\
\hline
\end{tabular}

Fuente: Elaboración propia a partir del Sistema de matricula de la Maestría en Gestión Educacional U.N.S.C.H-2019.

A los 28 docentes se les realizó entrevistas semi-estructuradas y a partir del fenómeno de saturación se seleccionó a 5 docentes quienes aportaron datos más resaltantes e información rica para su análisis (Jansen, 2012), considerándolos como informantes clave que mostraron 
ganas de participar y responder a las preguntas durante los encuentros, los cuales se les identificó con letras ( $A, B, C, D$ y E).

El cuestionario y las entrevistas fueron realizadas vía online, el cuestionario por Google forms y las entrevistas por medio de Zoom, dichos encuentros fueron grabados, durando una hora cada dos días a la semana, durante los meses de octubre, noviembre y diciembre del 2020, creando fichas para el registro de archivos de información para ser transcritas lo más rápido posible, donde el diálogo y la interpretación permitieron comprender los testimonios textuales. Para interpretar la información generada se aprovechó de las ventajas que brinda el programa ATLAS.ti, delineado por Mühr y Friese (2004), como mecanismo computacional para analizar información cualitativa de las declaraciones textuales.

\section{Percepciones de la innovación educativa y la práctica pedagógica en instituciones rurales}

Una vez aplicado el cuestionario sobre la innovación y práctica pedagógica la interpretación de la información mostrada en el gráfico 1, posibilita identificar que solo $50 \%$ de los docentes reconocen y perciben que la innovación educativa mejora la práctica pedagógica, el $32 \%$ medianamente reconocen que la innovación educativa mejora los aprendizajes de los estudiantes. Mientras que el $18 \%$ no reconocen que la innovación educativa por emergencia es la mejor oportunidad para generar cambios en las prácticas pedagógicas. Los docentes reconocen desde su percepción que la innovación educativa produce cambios y mejoras, sin embargo, en este tiempo de emergencia no lo ven como oportunidad para mejorar la práctica pedagógica, aun cuando todos han desarrollado actividades a través de las TIC que les ha permitido crecer académica y profesionalmente.

Gráfico 1. Percepciones sobre la innovación educativa y la práctica pedagógica

\begin{tabular}{|c|c|c|c|}
\hline $\begin{array}{c}\text { Innovación educativa por emergencia } \\
\text { como oportunidad de cambio }\end{array}$ & 18 & & \\
\hline $\begin{array}{c}\text { Innovación educativa para mejorar los } \\
\text { aprendizajes de los estudiantes }\end{array}$ & & 32 & \\
\hline $\begin{array}{c}\text { Innnovación educativa como mejora de la } \\
\text { práctica pedagógica }\end{array}$ & & & \\
\hline & 0 & 20 & 40 \\
\hline
\end{tabular}

Fuente: Elaboración propia.

En este estudio además de conocer algunas percepciones de los docentes que laboran en instituciones rurales se partió también de información valiosa mediante las entrevistas realizadas que le otorgan fiabilidad a la investigación. Todo esto con la finalidad de poder generar categorías y sub-categorías que emergieron como resultado de la interpretación de la información, centrándose en el análisis y síntesis para construir una percepción clara de la innovación educativa y práctica pedagógica docente en instituciones educativas rurales en 
Innovación educativa y práctica pedagógica docente en instituciones educativas rurales en el Perú en tiempos de pandemia

tiempos de pandemia. En la tabla 2 y Gráfico 2, se presentan en forma simplificada todas las categorías y sub-categorías adquiridas a través del programa ATLAS.ti

Tabla 2. Categorías y sub-categorías emergidas del programa ATLAS.ti

\begin{tabular}{|l|l|}
\hline \multicolumn{1}{|c|}{ Categorías } & \multicolumn{1}{c|}{ Subcategorías } \\
\hline Adaptación a la innovación educativa & Virtualidad \\
& Nuevas tecnologías \\
& Nuevos entornos de enseñanza aprendizaje \\
\hline Formación docente & Formación continua \\
& Práctica docente \\
& Pedagogía \\
& Didáctica \\
& Desempeño profesional \\
& Reprofesionalización \\
& Actualización \\
\hline Apoyo familiar & Comunicación e interacción \\
& Acompañamiento \\
\hline Nuevos roles de los docentes y estudiantes & Relación personalizada \\
& Activo \\
\hline & Dinámico \\
\hline
\end{tabular}

Fuente: Elaboración propia

Gráfico 2. Red de categorías

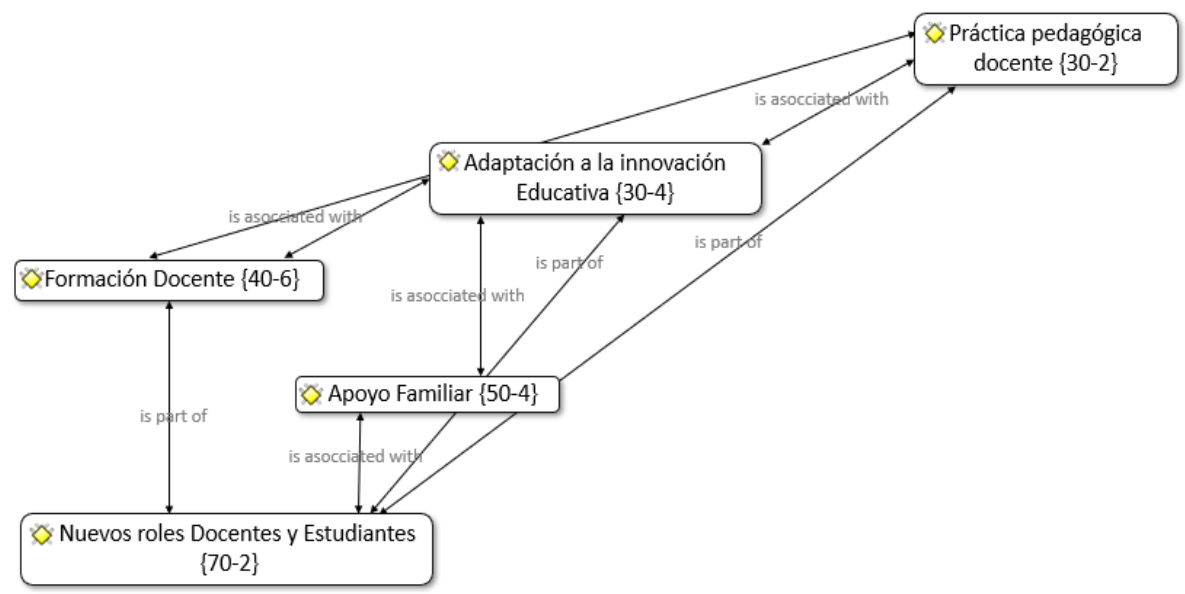

Fuente: Elaboración propia

La práctica pedagógica docente se evidencia en el desarrollo de las acciones y el discurso dentro del escenario escolar, en el pleno proceso formativo donde se integra no solo las 
relaciones con los estudiantes sino también los conocimientos que estos le aportan para su crecimiento personal. Comprender la nueva realidad escolar que cada día requiere del empleo de herramientas didácticas en ambientes virtuales para proporcionar la formación de los educandos en este tiempo de incertidumbre, es pertinente la ejecución de actividades individuales y en grupo con el objeto de desarrollar en ellos el trabajo autónomo, aprendiendo de otros y para otros (García Aretio, 2017).

La práctica docente bajo la modalidad a distancia necesita de variados elementos como la adaptación a las ideas nuevas, manejo de medios y recursos tecnológicos adecuados y al alcance de los educandos, formación continua, apoyo familiar, que han desencadenado nuevas formas de actuar, nuevas funciones y roles mediado por las tecnologías. Esto refleja una renovación y cambio en el papel del docente en el contexto escolar (Martínez-Garcés y GarcésFuenmayor, 2020).

\section{Adaptación a la innovación educativa}

La categoría adaptación a la innovación educativa se centra en el docente como una de las piezas fundamentales para que la innovación educativa y la práctica pedagógica en las instituciones educativas rurales en estos tiempos de pandemia se logre. Primero porque es un hecho que la educación a distancia o virtual vino para quedarse, y es evidente que ha marcado un antes y un después en el desempeño y la práctica docente. Además de mostrar la precariedad del sistema educativo y las desigualdades culturales, sociales y económicas en nuestro continente (Expósito y Marsollier, 2021). No obstante, la adaptación a la innovación educativa facilitó el desarrollo de la educación continua garantizando el derecho a educarse a los educandos tanto en el contexto rural como el urbano (Amuchástegui et al., 2017).

En el caso del contexto rural, la innovación educativa es una oportunidad de cambio para el docente y el sistema en general, dado que el sector red imperceptible y poco tomado en cuenta en la política peruana (Gómez-Arteta y Escobar-Mamani, 2021), debe ser considerado como una propuesta para las comunicaciones y oportunidad para lograr la adaptación a la innovación, las nuevas tecnologías y los nuevos entornos de aprendizaje; aunque fue con la pandemia que de manera forzada y drástica por la emergencia sanitaria vivida, ha tenido que responder mediante la readaptación de las actividades escolares presenciales a las virtuales (Cabrera, 2020), sin la adecuada capacitación, recursos y apoyo (UNESCO, 2020).

Los docentes ante esto percibieron durante varios meses que era todo un desafío adaptarse a emplear el computador y el móvil como medio de comunicación y herramienta de trabajo constante, algo que era eventual cuando el contenido lo ameritaba (Docente A y D). Mientras que otros docentes refirieron que no tenían computador y móvil con las tecnologías necesarias para dar clases, así mismo los niños, niñas y jóvenes tampoco poseen esto por su bajo nivel socioeconómico (Docentes $\mathrm{B}, \mathrm{C}$ y $\mathrm{E}$ ), lo que demuestra que los recursos fueron uno de los primeros elementos que limitaban a la adaptabilidad a la innovación educativa de los docentes y estudiantes del contexto rural.

Gracias a la estrategia generada por el Ministerio de Educación, quien ejecutó un programa denominado "Aprendo en casa" que ofreció clases por radio y televisión, así como las plataformas virtuales, que sirvieron de apoyo a la virtualización de la enseñanza aprendizaje posibilitó llegar a más lugares. Efectivamente la adaptación a la virtualización educativa en contextos rurales ha mantenido a la educación de cientos de niños y jóvenes que requieren ser 
Innovación educativa y práctica pedagógica docente en instituciones educativas rurales en el Perú en tiempos de pandemia

formados, y sus docentes han ido construyendo sus propios aprendizajes, siendo responsables con los educandos a enseñar a manejarse en este entorno, en función del medio digital que esté al alcance de los educandos (Bonilla-Guachamín, 2020; Dussel, 2020).

Si bien es cierto, es bueno resaltar que a pesar de los bajos recursos que poseen las instituciones educativas rurales, tal como lo expresan los informantes era un gran desafío para ellos adaptarse pues en su mayoría los encuentros eran presenciales, y la innovación a través de las TIC, eran discretas poco usada, por los que las experiencias con ellas eran aisladas y complementaria como estrategia innovadora (Almirón y Porro, 2014). Los docentes de las instituciones rurales, a pesar de la poca conexión a internet (González y Cabrera, 2010; Dussel, 2020), han sabido salir adelante, ante estos tiempos de incertidumbre, incorporando los medios digitales disponibles en la realización de las actividades escolares.

En la medida que el docente comprenda que la sociedad postmoderna está en una dimensión temporal cambiante este debe ir readaptándose a la innovación educativa, debido a que los educandos aprenderán al ritmo en lo que son enseñados generando transformaciones en sus percepciones sociales y culturales, por lo que el docente debe dejar a un lado la resistencia al cambio y evolucionar en su práctica pedagógica (Perrenoud, 2004; Molina Pacheco y Mesa Jiménez, 2018).

\section{Formación Docente}

Para dar paso a las innovaciones educativas como saber manejar el computador, el móvil o cualquier equipo que apoye a las telecomunicaciones, así como romper la concepción de educar solo en espacios físicos fijos, y que este proceso pueda darse en tiempo real 0 programados desde cualquier parte y a cualquier hora, es un gigantesco paso para el sistema educativo de la educación básica peruana. Esto lo permitió la pandemia que, si bien esta situación ha ocasionado graves problemas de salud, económicos y educativos, ha traído beneficios desde el panorama escolar para que el docente en su práctica pedagógica, renueve su acción, lo que ha conllevado a actualizarse, reprofesionalizarse emplear una pedagogía y didáctica diferente mejorando su desempeño profesional.

Por lo que el docente ha tenido la necesidad de capacitarse y adquirir competencias digitales en unas cuantas semanas para establecer la comunicación y colaboración entre educandos, docentes y familiares (González Fernández, 2021). Este proceso de virtualización del aprendizaje ha sido un desafío pedagógico y asunto complejo (De Luca, 2020). Tal es así, que algunos docentes manifestaron sentirse incompetentes, fracasados ante el manejo de herramientas tecnológicas y medios colaborativos destacando no adaptarse a ellas (Docente $\mathrm{B}$ y E); no obstante, otros señalaron que, aunque es diferente el hacer están dispuestos a autoformarse, capacitarse, dedicar más tiempo y aprovechar todo lo que ofrecen los estudios planteados por la dirección escolar y currículas universitarias que les ayude a apropiarse de competencias pedagógicas digitales para la mejora de su práctica en clase (Docente A, C y D).

Lo que muestra que todos los docentes que laboran en instituciones rurales necesitan formarse y actualizarse para adquirir competencias digitales y adaptarse a las condiciones que estamos viviendo (Díaz Hoyos et al., 2020; Flores Peña y Navarrete Cueto, 2020; Picón et al., 2020). Las habilidades y capacidades para asumir la práctica en el aula ante la pandemia eran 
diferentes, por los cuales son insuficientes para enfrentar la educación a distancia, debido a que no estaban preparados para aceptarla.

Por lo que coincide con lo planteado por Sánchez Mendiola et al. (2020), al señalar que el papel del docente cambia de una perspectiva tradicional a una innovadora digital donde este dirige ambientes que induce al educando a adquirir conocimientos de manera constructiva y dinámica acompañando al aprendiz en todo el proceso ya sea en ambientes sincrónicos 0 asincrónicos. El hecho de ser un buen docente en la educación presencial no garantiza el mismo desempeño cuando se desenvuelve en ambientes virtuales (Gros Salvat y Silva Quiroz, 2005). Esto lleva a que el docente reflexione sobre los mecanismos de aprender a enseñar y potenciar el autoaprendizaje en estos contextos, mediante su formación continua (Suárez, 2020).

Por todo esto se puede inferir que, a partir de la autoformación docente, capacitación y actualización, ha aumentado la actitud del profesorado con respecto al reconocimiento de la innovación educativa por emergencia como la mejor oportunidad para generar cambios en las prácticas pedagógicas, dado que una minoraría de los participantes no la aprecian como tal, mientras que la mayoría de los informantes han percibido los avances en la integración de la tecnología, al reconocer que la innovación educativa mejora la práctica pedagógica y los aprendizajes de los estudiantes.

Esta actitud y percepción ha marcado que las experiencias tanto favorables como desfavorables adquiridas en la práctica ha facilitado la adquisición de algunas competencias digitales comprendiendo así su rol como docente virtual (Gros Salvat y Silva, Quiroz 2005). Esto ha conducido el cambio de percepción por medio de la autocapacitación de muchos docentes que antes de la pandemia se resistían al desarrollo de las competencias digitales y se vieron forzados a experimentarlas y apropiarse de ellas para ver resultados académicos en sus educandos (Padilla Partida, 2018; Martínez-Garcés y Garcés-Fuenmayor, 2020).

\section{Apoyo familiar}

Esta categoría es muy importante; el apoyo familiar ha sido moderado bajo, por las precariedades en la que vive la zona rural del Perú, por su conocida brecha de desigualdad social, que se recrudeció en estos tiempos de pandemia (Gómez-Arteta y Escobar-Mamani, 2021). La falta de equipos, la inestable conexión a internet, y otras telecomunicaciones como la radio y la televisión mediante la estrategia "Aprendo en casa" han mitigado de alguna manera el proceso de enseñanza aprendizaje. Sin embargo, la innovación educativa a través de la modalidad virtual generó varias contrariedades, principalmente la comunicación con los padres de familia, quienes trabajan y son analfabetos, por lo que los estudiantes no reciben retroalimentación o refuerzo en el hogar. Además, que los educandos no tienen los recursos para ver las guías, por lo que se le dificulta al docente hacer el acompañamiento, orientación y realización de las actividades propuesta en el programa escolar (Aragón y Cruz, 2020).

Al respecto, esto corresponde con lo manifestado por los docentes entrevistados quienes respondieron que se les dificulta establecer comunicación recurrente con sus educandos y padres de familia, por lo que muchas veces deben ir a su encuentro en zonas amplias y lejanas como cerros, a impartir las clases con las medidas de distanciamiento requeridas, trabajando con los educandos con teléfono y los que no tienen escuchan la realimentación realizada por el docente y el resto de sus compañeros (Docente B,C y E); por otro lado, los que poseen teléfono, computadora, radio o televisión, reciben clases por mensajería a través del Messenger o 
Innovación educativa y práctica pedagógica docente en instituciones educativas rurales en el Perú en tiempos de pandemia

WhatsApp, quienes reciben el acompañamiento a distancia, son evaluados con actividades que deben ser adjuntas a sus correos para observar y corregir el proceso (Docente A, B, C, D y E); este grupo de estudiantes que tiene acceso a internet y recursos es muy reducido, por lo que poseen ventajas en la interacción y el desarrollo de varias habilidades y competencias digitales.

Estas situaciones, aunque son diferentes benefician a unos y desfavorecen a otros; es de considerar para hacer una profunda reflexión pues acentúa las desigualdades sociales y culturales (Tarabini, 2020; Vivanco-Saraguro 2020), donde los docentes y educandos todavía no se encuentran a tope con la formación requerida para dar respuestas ante las crecientes demandas en el manejo de las TIC. Así mismo, es urgente que existan políticas educativas que se extienda después de la pandemia, que fortalezca la educación virtual en todos los niveles para poder asumirla, accederla y sacar provecho de ella (Expósito y Marsollier, 2021).

\section{Nuevos roles de los docentes y estudiantes}

Es evidente que los roles de los docentes y educandos han cambiado; en los docentes la relación debe ser ampliamente personalizada, flexible, donde debe manejar equipos nuevos y sus aplicaciones para la comunicación e interacción, que promueva una enseñanza activa y dinámica que fortalezca los aprendizajes de los educandos (García-García, 2020). Donde lo pedagógico y su práctica se fortaleció tal como lo expresan los Docentes $A$ y $D$, al referir que convirtieron la enseñanza presencial a enseñanza virtual o remota desde su hogar, influyendo con los materiales didácticos a la mano con el objeto de que los educandos comprendieran los temas, haciendo que este sea constructivo, activo y crítico. Así como también, motivadores de vida, promotores de superación de circunstancias dolorosas, porque muchos de los estudiantes $y$ docentes sufrieron pérdidas de seres queridos (Docentes $\mathrm{B}, \mathrm{C}, \mathrm{E}$ ).

Esto demuestra una vez más que la innovación educativa y la práctica pedagógica docente en instituciones educativas rurales en tiempos de pandemia, fue más allá de lo escolar, tal como lo afirma Villafuerte et al. (2020), fue hacia la contención afectiva y emocional, asumiendo un rol de centinela de la esperanza al progreso de la educación. Es preciso destacar, que el nuevo rol del docente es seguir haciendo que el educando se sienta protagonista de su propio aprendizaje, que tiene un rol importante que debe asumir dentro del proceso de enseñanza aprendizaje virtual que solo se logra mediante la autodisciplina para autodirigir sus capacidades y adquirir los conocimientos propio de su nivel (Rugeles Contreras et al., 2015). Con estos nuevos roles de los docentes y educandos mediados por las TIC, no solo se generará nuevos conocimientos, sino que se promoverá y fortalecerá competencias pedagógicas y digitales en los principales actores educativos tan relevantes para hacer frente a los retos del futuro.

Es por ello, que el educando del contexto rural a pesar de las pocas oportunidades y dificultades de acceso a las TIC, desarrollaron un rol en la virtualidad más comunicativo y colaborativo, siendo una figura activa de su aprendizaje, crítico ante lo que lee y disfruta, debido a que se motiva por las múltiples formas de aprender a través del acceso actualizado de información, flexibilidad de revisar en el momento que considere necesario e interacción sincrónica y asincrónica con sus pares, así como conocer infinidades de zonas geográficas y culturales, sin límite alguno (Suárez y Najar, 2014; Salgado et al., 2017). 


\section{Conclusiones}

La expansión del COVID-19, ha afectado todos los sectores productivos, especialmente el educativo, generando transformaciones desde la modalidad presencial a la virtual, esa que se puede acceder a distancia desde cualquier parte donde se encuentren. Sin embargo, desde los escenarios educativos rurales existen ciertas limitantes como falta de apoyo familiar, fallas de conexión constante que dificultan en la mayoría de las veces llevar a cabo las clases online, por lo que pudiera desmotivar al estudiante.

De acuerdo a la percepción de los docentes entrevistados la innovación educativa y práctica pedagógica en el sector rural al principio se tornó difícil, pues cayó de sorpresa, sin planificación, ni formación sobre una modalidad poco conocida y experimentada por la mayoría de docentes y educandos como un modelo educativo que diera respuestas al desarrollo de los aprendizajes; más bien era vista desde la óptica del entretenimiento, comunicación y distracción.

Por lo que el docente se adaptó a la situación, al nuevo escenario escolar asumiendo que era necesario aprender, autoformarse y actualizarse para el manejo de los equipos y las aplicaciones y hacer su práctica pedagógica desde su hogar, por lo incierto del panorama, su formación no fue la más adecuada, pero gracias a las pocas habilidades del docente, se transformó la enseñanza presencial a la enseñanza virtual que ha tenido tropiezos, pues aún persisten muchas debilidades en la enseñanza virtual, sin discutir las bondades que ha traído su apoyo al proceso de aprendizaje de los educandos.

Hay cambios de percepciones y roles de los principales actores educativos, percepciones favorables; que evidentemente los aprendizajes para el docente y el educando no es el mismo, por lo que el docente ha requerido capacitarse en el empleo de estrategias pedagógicas y didácticas para el manejo de recursos virtuales, lo que es considerado un gran avance para reducir brechas socioeducativas y culturales. Asimismo, el establecimiento de nuevas formas de relacionarse, fortaleciendo lazos entre docentes y estudiantes, dándole continuidad a los procesos educativos. Por lo que es necesario desde la dirección escolar, desarrollar acciones sostenidas centradas en las TIC para la capacitación docente, planificación gestión y disposición de recursos con el objeto de ofrecer mejores experiencias educativas.

\section{Referencias Bibliográficas}

Almirón, Miriam., y Porro, Silvia. (2014). Los docentes en la Sociedad de la Información: reconfiguración de roles y nuevas problemáticas. IE Comunicaciones. Revista Iberoamericana de Informática Educativa, Vol. 19, España. (Pp.17-31). https://dialnet.unirioja.es/servlet/articulo?codigo $=4794547$

Amuchástegui, Griselda., Del Valle, Maria Isabel., y Renna, Henry. (2017). Reconstruir sin ladrillos: guías de apoyo para el sector educativo en contextos de emergencia. http://repositorio.minedu.gob.pe/handle/MINEDU/5592

Aragón, Jorge., y Cruz, Marylia. (2020). 2020: el año de las maestras y maestros en el Perú. Escuela de Gobierno y Políticas Públicas. Recuperado de https://escuela.pucp.edu.pe/gobierno/investigacion/reportes-tematicos-2/2020-el-anode-las-maestras-y-maestros-en-el-perul.

Basto-Torrado, Sandra Patricia. (2011). De las concepciones a las practicas pedagógicas de un grupo de profesores universitarios. Magis, Revista internacional de investigación en 
Innovación educativa y práctica pedagógica docente en instituciones educativas rurales en el Perú en tiempos de pandemia
educación,
Vol.3
núm, 6 ,
Colombia.

(Pp.393-412).

http://www.redalyc.org/articulo.oa?id=281021734009

Bonilla-Guachamín, Johana Alexandra. (2020). Las dos caras de la educación en el COVID-19.
CienciAmérica,
Vol.9,
núm.
2, Ecuador.
(Pp.89-98).

http://dx.doi.org/10.33210/ca.v9i2.294

Cabrera, Leopoldo. (2020). Efectos del coronavirus en el sistema de enseñanza: aumenta la desigualdad de oportunidades educativas en España. Revista de Sociología de la Educación-RASE, Vol. 13, núm. 2, España. (Pp.114-139). https://doi.org/10.7203/RASE.13.2.17125

De Luca, Marina Patricia. (2020). Las aulas virtuales en la formación docente como estrategia de continuidad pedagógica en tiempos de pandemia. Usos y paradojas. Análisis Carolina, Vol.33, España. (Pp.1-12). https://www.fundacioncarolina.es/wpcontent/uploads/2020/06/AC-33.-2020.pdf

Díaz Hoyos, Juan Alfredo., Sánchez Sánchez, María Julia., Aguilera Rodríguez, Maribel Evelyn., Loyola Polo, Kiko Edinson., Ramírez Castro, José Antonio. y Reynosa Navarro, Enaidy. (2020). Capacitación docente y calidad educativa en tiempos de COVID-19. Revista Científica, Cultura, Comunicación y Desarrollo, Vol. 5, núm.3, Cuba. (Pp.84-89). https://rccd.ucf.edu.cu/index.php/aes/article/view/263/290

Dussel, Inés. (2020). La formación docente y los desafíos de la pandemia. Revista científica EFI.DGES, Vol.6, núm.10, Argentina. (Pp.11-25). http://dges-cba.edu.ar/wp/wpcontent/uploads/2020/08/Dussel.pdf

Estrada Araoz, Edwin Gustavo., y Mamani Roque, Maribel. (2021). Competencia digital y variables sociodemográficas en docentes peruanos de educación básica regular. Revista San Gregorio. 45. Ecuador. (Pp. 1-16). http://scielo.senescyt.gob.ec/pdf/rsan/v1n45/2528-7907-rsan-1-45-00001.pdf

Expósito, Cristián David y Marsollier, Roxana Graciela. (2021). Virtualidad y educación en tiempos de COVID-19. Un estudio empírico en Argentina. Educación y Humanismo, Vol.22, núm.39, Colombia. (Pp.1-22). https://doi.org/10.17081/eduhum.22.39.4214

Flores Peña, Maira Rosalía., y Navarrete Cueto, Carlos Antonio. (2020). Diagnóstico de necesidades de capacitación en el uso de plataformas virtuales ante la contingencia del COVID-19 en los estudiantes y docentes de Educación Media Superior Tecnológica. Dilemas Contemporáneos: Educación, Política y Valores, Vol. 8, núm. 5. México. (Pp.1-21). https://doi.org/10.46377/dilemas.v8i.2494

Gadamer, Hans-Georg. (1993). Título original: WahrheitundMethode. Tradujeron: Ana Agud Aparicio y Rafael de Agapito. J.C.H., Mohr (Paul Siebeck) Tübingen. Traducido: Verdad y método. Fundamentos de una hermenéutica filosófica. Ediciones Sígueme, S.A. (quinta edición). España.

García Aretio, Lorenzo. (2017). Educación a distancia y virtual: calidad, disrupción, aprendizajes adaptativo y móvil. RIED. Revista Iberoamericana de Educación a Distancia, Vol. 20, núm, 2, España (Pp. 9-25. http://www.redalyc.org/articulo.oa?id=331453132001

García-García, María Daniela. (2020). La docencia desde el hogar. Una alternativa necesaria en tiempos del COVID 19. Polo del Conocimiento: Revista científico-Académica 
Multidisciplinaria, Vol.5, núm,4, Ecuador. (Pp. 304-324). https://dialnet.unirioja.es/servlet/articulo?codigo=7398376

Gómez-Arteta, Indira y Escobar-Mamani, Fortunato. (2021). Educación Virtual en tiempos de pandemia: incremento de la desigualdad social en el Perú. Chakiñan, Revista De Ciencias Sociales y Humanidades. PREPRINT PAPERS. Ecuador. (Pp. 1-12). Recuperado a partir

https://chakinan.unach.edu.ec/index.php/chakinan/article/view/553

González Fernández, María Obdulia. (2021). La capacitación docente para una educación remota de emergencia por la pandemia de la COVID-19. Revista Tecnología, Ciencia y Educación, Vol.19, España. (Pp.81-102). https://doi.org/10.51302/tce.2021.614

González, Wilder y Cabrera, Isaac. (2010). Educación virtual, conectividad y desigualdades: eduWeb una alternativa para la publicación de web docentes en el posgrado. Revista Electrónica Actualidades Investigativas en Educación, Vol.10, núm. 3, Costa Rica. (Pp.1-23). https://revistas.ucr.ac.cr/index.php/aie/article/view/10147

Gros Salvat, Begoña., y Silva Quiroz, Juan. (2005). La formación del profesorado como docentes en los espacios virtuales de aprendizaje. Revista Iberoamericana de Educación, Vol. 36, núm,1, España. (Pp. 1-13). https://doi.org/10.35362/rie3612831

Heidegger, Martin. (2003). Ser y tiempo. Editorial Fondo de Cultura Económica. Editorial Trotta, España.

Hernández Barrios, Yisel., Fonte Galindo, L.uis, Zabala Argüelles, Marìa., \& Pérez Chacón, Dennis. (2021). Mejorando la respuesta a la COVID-19: reorientación de los esfuerzos de comunicación de riesgos hacia cuestiones de equidad. Revista Latinoamericana De

Difusión Científica, Vol. 3, núm. 5, (Pp.3-8). https://doi.org/10.38186/difcie.35.01

Hernández Pino, Yoli Marcela. (2015). Factores que favorecen la innovación educativa con el uso de la tecnología: una perspectiva desde el proyecto coKREA. Revista Virtual Universidad Católica del Norte, Vol. 45, Colombia. (Pp. 38-52). Recuperado de http://revistavirtual.ucn.edu.co/index.php/RevistaUCN/article/ view/654/1187

Hernández-Sampieri, Roberto., y Mendoza, Cristian. (2018). Metodología de la investigación.

Las rutas cuantitativa, cualitativa y mixta. Editorial Mc Graw Hill Education. México.

Islas, Claudia. (2018). Implicación de las TIC en el aprendizaje de los universitarios: una explicación sistémico-conectivista. Pixel-Bit, Revista de Medios y Educación, Vol.52, España. (Pp.199-215). https://recyt.fecyt.es/index.php/pixel/article/view/62532

Jansen, Harrie. (2012). La lógica de la investigación por encuesta cualitativa y su posición en el campo de los métodos de investigación social. Paradigmas: Una Revista Disciplinar de Investigación. Vol. 5, Núm. 1. España. (Pp. 39-72). Extraído de: https://dialnet.unirioja.es/descarga/articulo/4531575.pdf

Loaiza Zuluaga, Yasaldez Eder., Rodríguez Rengifo, Juan Carlos., Vargas López, Hernán Humberto. (2012). La práctica pedagógica de los docentes universitarios en el área de la salud y su relación con el desempeño académico. Revista Latinoamericana de Estudios Educativos, Vol. 8, núm. 1, Colombia (Pp. 95- 118). Disponible en: http://www.redalyc.org/articulo.oa?id=134129256006

Martínez-Garcés, Josnel., y Garcés-Fuenmayor, Jackeline. (2020). Competencias digitales docentes y el reto de la educación virtual derivado de la covid-19. Educación y 
Innovación educativa y práctica pedagógica docente en instituciones educativas rurales en el Perú en tiempos de pandemia
Humanismo.
Vol.
22, núm,39,
Colombia
(Pp.
$1-$

16). https://doi.org/10.17081/eduhum.22.39.4114

Méndez Calderón, Margelis Coromoto. (2021). Covid-19 un fenómeno colectivo, visto desde el umbral de la biopolítica. Revista Latinoamericana De Difusión Científica, Vol.3. núm.5, Venezuela. (Pp.25-35). https://doi.org/10.38186/difcie.35.03

Molina Pacheco, Luis Eduardo y Mesa Jiménez, Fredy Yesid. (2018). Las tic en Escuelas Rurales: realidades y proyección para la Integración. Praxis \& Saber, Vol. 9, núm. 21, Colombia. (Pp. 75-98. https://www.redalyc.org/journal/4772/477258898004/html/ Moreno-Correa, Sandra Milena. (2020). La innovación educativa en los tiempos del Coronavirus. Salutem Scientia Spiritus, Vol. 6, núm. 1. Colombia. (Pp.14-26). https://revistas.javerianacali.edu.co/index.php/salutemscientiaspiritus/article/view/2290

Mühr, Thomas y Friese, Susanne. (2004). Manual de usuario para ATLAS.ti. Versión 7.5.4, (2nd Edición). Desarrollo de software científico. Alemania Extraído de: https://doc.atlasti.com/ManualWin.v9/ATLAS.ti_ManualWin.v9.pdf.

Neira-González, María Fernanda., \& Pulgarin, Erwin Estuardo. (2021). La Innovación Educativa como herramienta pedagógica en el proceso de enseñanza-aprendizaje en tiempos de pandemia de la Unidad Educativa Fiscal José Jesús Ocampo Salazar. Digital Publisher CEIT, Vol.6, núm.1, Ecuador. (Pp.96-120). https://doi. org/10.33386/593dp.2021.1.425

Organización Mundial de la Salud (OMS). (2020). Cronología de la actuación de la OMS: https://www.who.int/es/news-room/detail/27-04-2020-who-timeline---covid-19

Orozco-Cazco, Gustavo., Cabezas-Gonzàlez, Marcos., Martínez-Abad, Fernando, y Abaunza, Geovanny. (2020). Variables sociodemográficas que inciden en las competencias digitales del profesorado universitario. Chakiñan, Revista de Ciencias Sociales y Humanidades, 12, Ecuador. (Pp.32-48). https://doi.org/10.37135/chk.002.12.02

Padilla Partida, Siria. (2018). Usos y actitudes de los formadores de docentes ante las TIC. Entre lo recomendable y la realidad de las aulas. Apertura, Vol. 10, núm.1, México. (Pp.132148). http:// dx.doi.org/10.32870/Ap.v10n1.1107

Parra, Jorge E., y Méndez, Marlon. (2005). Pedagogía y educación virtual en un programa de extensión rural. Agronomía Colombiana, Vol. 23, núm. 1, Colombia. (Pp. 171-182). Disponible en: http://www.redalyc.org/articulo.oa?id=180316951021

Peirano R, Claudia., Estévez S, Swapna Puni y Astorga, María Isabel. (2015). Educación rural: oportunidades para la innovación. Cuadernos de Investigación Educativa, Vol. 6, núm.

1, Uruguay (Pp. 53-70). http://www.scielo.edu.uy/pdf/cie/v6n1/v6n1a04.pdf

Perrenoud, Phillipe. (2004). Desarrollar la práctica reflexiva en el centro de enseñanza. Barcelona: Grao.

Picón, Gerardo., González de Caballero, Gricelda., y Paredes, Juana. (2020). Desempeño y formación docente en competencias digitales en clases no presenciales durante la pandemia COVID-19. Human Sciences.

https://preprints.scielo.org/index.php/scielo/preprint/view/778/1075

Pila Martínez, Juan Carlos., Andagoya Pazmiño, Wuilson Gonzalo., \& Fuertes Fuertes, María Elizabeth. (2020). El profesorado: Un factor clave en la innovación educativa. Revista EDUCARE-UPEL-IPB - Segunda Nueva Etapa 2.0, Vol. 24, núm.2, Venezuela. (Pp.212232). https://doi.org/10.46498/reduipb.v24i2.1327 
Ramírez, Leticia. (2020). Tendencias de la innovación educativa en los contextos sociales. Análisis del mapeo de literatura. Revista Educación, Vol.44, núm 1, Costa Rica. (Pp.2215-2644). DOl: https://doi.org/10.15517/revedu.v44i1.33222

Romero Escalante, Víctor Fabian. (2020). Disrupción de la educación remota en el programa universitario para adultos en tiempos de covid-19. Apuntes Universitarios, Vol.11, núm,1, Perú. (Pp.401 - 413). https://doi.org/10.17162/au.v11i1.593

Rugeles Contreras, Paul Andrés., Mora González, Beatriz'., y Metaute Paniagua, Piedad María. (2015). El rol del estudiante en los ambientes educativos mediados por las TIC. Revista Lasallista de investigación. Vol. 12, núm. 2, Colombia. (Pp.132-138). https://www.redalyc.org/pdf/695/69542291025.pdf

Salgado, Efrén., Gómez, Marcela., y Pintor, María. (2017). Educación rural y la adquisición de competencias laborales: una innovación mediante el uso de recursos digitales. Educatio Siglo XXI, Vol.35, España. (Pp.33-54).https://doi.org/10.6018//286211

Sánchez Mendiola, Melchor., Martínez Hernández, Ana María., Torres Carrasco, Ruth., Agüero Servín, M. ${ }^{a}$ Mercedes de, Hernández Romo, Alan., Benavides Lara, Mario Alberto., Jaimes Vergara, C. A. y Rendón Cazales, V. J. (2020). Retos educativos durante la pandemia de COVID-19: una encuesta a profesores de la UNAM. Revista Digital Universitaria, Vol. 21, núm.3, México. (Pp.1-24). https://www.revista.unam.mx/wpcontent/uploads/AOP.pdf

Sandia, Beatriz., Aguilar, Alba., y Luzardo, Marianela. (2018). Competencias digitales de los docentes de educación superior. Caso Universidad de Los Andes. Educere, Vol.22, núm. 73, Venezuela. (Pp.603-616). Recuperado de: https://www.redalyc.org/journal/356/35656676011/

Sein-Echaluce, María Luisa., Fidalgo-Blanco, Angel., y Alves, Gustavo. (2016). Technology behaviors in education innovation. Computers in Human Behavior, In press. Vol. 30. EE.UU. (Pp.1-3). http://dx.doi.org/10.1016/i.chb.2016.11.049

Suárez, Noemí. (2020). Formación docente universitaria y crisis sanitaria COVID-19. CienciAmérica, Vol.9, núm.2, Ecuador. (Pp.109-114). Disponible en: http://cienciamerica.uti.edu.ec/openjournal/index.php/uti/article/view/299

Suárez, Nubia Esperanza., y Najar, José Custodio. (2014). Evolución de las tecnologías de información y comunicación en el proceso de enseñanza-aprendizaje. Revista vínculos, Vol.11, núm.1, Colombia.

(Pp.209-220).

https://revistas.udistrital.edu.co/index.php/vinculos/article/view/8028

Tarabini, Aina. (2020). ¿Para qué sirve la escuela? Reflexiones sociológicas en tiempos de pandemia global. Revista de Sociología de la Educación-RASE, Vol.13, núm. 2, España. (Pp.145-155). https://doi.org/10.7203/RASE.13.2.17135

Torres-González, José. (2021). Reprofesionalización docente e inclusión en tiempos de pandemia. Revista Internacional de Investigación Ciencias Sociales.Vol. 17 núm. 1, Paraguay. (Pp. 01-03). http://revistacientifica.uaa.edu.py/index.php/riics/article/view/991/pdf

UNESCO. (2020). Education: From disruption to recovery. Recuperado de https://en.unesco.org/covid19/educationresponse

Vásquez-Ponce, Gary Orlindo., Indacochea-Figueroa, Juleisy Fernanda., Forty-Moreira, Richard., Chara-Plúa, EillenJahel. (2020). Educación virtual en tiempos del covid-19 
desde la perspectiva socioeconómica de los estudiantes de la Universidad Estatal del Sur de Manabí del cantón Jipijapa. Polo del conocimiento. Vol. 5 núm. 10, Ecuador. (Pp.798-823). https://dialnet.unirioja.es/servlet/articulo?codigo=7659394

Vila Pérez, Olga., y Núñez García, Elieder. (2020). La práctica pedagógica con enfoque prospectivo en el sistema de escuelas del Partido. Revista Conrado, Vol.16, núm. 73, Cuba. (Pp.165-170).https://conrado.ucf.edu.cu/index.php/conrado/article/view/1288

Villafuerte, Jhonny., Bello, Jhoana., Cevallos, Yisela., y Bermello, Jinsop. (2020). Rol de los docentes ante la crisis del COVID-19, una mirada desde el enfoque humano. REFCalE: Revista Electrónica Formación y Calidad Educativa, Vol.8, núm.1, Ecuador. (Pp.134150). http://refcale.uleam.edu.ec/index.php/refcale/article/view/3214

Vivanco-Saraguro, Ángel. (2020). Teleducación en tiempos de COVID-19: brechas de desigualdad. CienciAmérica, Vol. 9, núm,2, Ecuador. (Pp.166-175). http://cienciamerica.uti.edu.ec/openjournal/index.php/uti/article/view/307

Watermeyer, Richard., Crick, Tom., Knight, Catrin., \& Goodall, Janet. (2021). COVID-19 and digital disruption in UK universities: afflictions and affordances of emergency online migration. High Educ. Vol. 81, EE.UU. (Pp. 623-641). https://doi.org/10.1007/s10734$\underline{020-00561-y}$ 